\title{
Respuestas a la audiencia con el Comité Asesor Presidencial de Políticas para la Infancia
}

\author{
FRANCISCO MORAGA M. ${ }^{1}$, CARLOS SAIEH A. ${ }^{2}$, ARNOLDO QUEZADA L. ${ }^{3}$ \\ 1. Servicio de Pediatría Hospital San Borja Arriarán, Director Sociedad Chilena de Pediatría. \\ 2. Clínica Las Condes. Director Sociedad Chilena de Pediatría. \\ 3. Departamento de Pediatría Sur. Facultad de Medicina Universidad de Chile, Hospital Exequiel González C. \\ Presidente Sociedad Chilena de Pediatría 2005-2006.
}

\begin{abstract}
Answers to the audience with the Government Advisory Committee on Infancy Politics

Presentation of the answers delivered by the Board of Directors from the Chilean Paediatrics Society (SOCHIPE) to the Government Advisory Committee on Infancy Politics, including the paediatrician view on epidemiologic and demographic changes, its role in "Development and Behaviour in Paediatrics" and performance in infant primary health attention. It analyses SOCHIPE participation in human resources and health reform, with special emphasis in GES pathologies and chronic diseases impact, in relation to the available resources for health care. Finally, it discusses SOCHIPE mission in increasing the genetic potential expression of children and the biopsychosocial view of medicine.

(Key words: infancy politics, paediatrician role, SOCHIPE role, health reform, primary health care, holistic vision).

Rev Chil Pediatr 2007; 78 (Supl 1): 51-56
\end{abstract}

\section{RESUMEN}

Se presentan las respuestas entregadas por el Directorio de la Sociedad Chilena de Pediatría (SOCHIPE) al Comité Asesor Presidencial para la Reforma de Políticas de Infancia, que incluyen la visión del pediatra en el escenario de los cambios epidemiológicos y demográficos del nuevo milenio, su rol en la llamada "Pediatría del comportamiento y del desarrollo" y la participación de la atención primaria en salud infantil. Se comenta y analiza la participación de SOCHIPE en la formación de recursos humanos, en la reforma de salud en marcha, con especial énfasis en el impacto de las patologías con Garantías Explícitas (GES) y la atención de niños con enfermedades crónicas, en relación con los recursos disponibles para la atención de salud. Finalmente se discute la misión de SOCHIPE sobre el afianzamiento de la expresión del potencial genético de todos los niños y la visión biopsicosocial de la medicina.

(Palabras clave: Políticas de infancia, rol del pediatra, rol de la Sociedad Chilena de Pediatría, reforma de salud, atención primaria en salud, visión holística).

Rev Chil Pediatr 2007; 78 (Supl 1): 51-56 


\section{Introducción}

En el mes de Mayo del año recién pasado, la Sociedad Chilena de Pediatría, representada por su Presidente, fue recibida en audiencia por el Comité Asesor Presidencial para la Reforma de las Políticas de Infancia. Luego de la presentación sobre la situación actual de la salud infantil, cuyo contenido corresponde al documento "Epidemiología, nueva morbilidad pediátrica y rol del pediatra" contenido en este mismo número, los integrantes de esa comisión plantearon varias interrogantes que se analizan en este documento y que hemos decidido publicar por cuanto consideramos que su contenido es de interés general.

\section{Visión del pediatra en el nuevo milenio}

La Pediatría, más que una especialidad, puede considerarse una disciplina integradora que ve, estudia y atiende al ser humano en su proceso de desarrollo. La Pediatría es la medicina del desarrollo humano y tiene, por lo tanto, un significado, un alcance y una proyección enorme y de múltiple trascendencia. El desarrollo humano es -y debe ser- el componente fundamental y principal del progreso social. Por más adelantos científicos que la inventiva humana nos muestre diariamente, sin desarrollo humano pleno, armónico e integral y sin que esos adelantos beneficien por igual a todos los hombres, no puede hablarse de un auténtico progreso social.

El pediatra es el profesional que está más próximo a las primeras etapas del ciclo vital de cada ser humano y el que puede observar e intervenir en su proceso de crecimiento y desarrollo; en cada niño que atienda tiene que ver al continuador de una generación humana, al realizador de un proyecto histórico humano. Es el profesional de salud que tiene más posibilidades de influir en el niño, en la familia, en la colectividad, en la sociedad, en su cultura, en el presente y fundamentalmente, en el futuro. Su comprensión de la salud y su quehacer no pueden quedar limitados en la labor curativa o rehabilitadora sino que debe extenderse a la promoción y prevención. No se trata de actuar sólo ante enfermedades o riesgos visibles para el desarrollo infantil, sino propiciar las mejores condiciones familiares y sociales para éste. $\mathrm{Su}$ responsabilidad, por lo tanto, no se limita a la atención en la consulta o la hospitalización, sino que se extiende a la conducción y orientación de personas, equipos y redes médico sociales para promover el desarrollo integral del niño.

La promoción del desarrollo integral del niño implica el reconocimiento de la salud como un derecho universal e irrestricto (no como una necesidad o un bien transable), la defensa de la igualdad de oportunidades para todos los niños, reformular los estilos actuales de hospitalización y tratamiento ambulatorios y adherirse a las actividades en promoción de la vida. En tal sentido, a los pediatras les compete la responsabilidad de contribuir a fomentar una cultura de la salud y el desarrollo, así como también ser inductor de una conducta ética activa al interior de los equipos de salud, de las sociedades científicas y de los niveles de decisión estatal sobre lo que se está haciendo o dejando de hacer por los niños de nuestro país. La situación de la niñez en países como los nuestros es compleja: a los males derivados de la pobreza y la estructuración social, se suman los de la insuficiente atención de la salud pública. Sin embargo, hay pocas instituciones que dejan oír su voz en defensa de la salud y el desarrollo infantil. ¿Estamos los pediatras, como cuerpo profesional competente y autorizado, sintonizados con ellos? ¿Se escucha nuestra voz de manera constante a favor de los niños, en apoyo a sus esfuerzos por conquistar la salud y una calidad de vida mejor?. No basta una sólida y actualizada formación académica permanente, fundamental para el enfrentamiento de los problemas del niño enfermo o sano, sino que se requiere de una acabada formación humanista e integral que nos permita comprender todo el panorama de la niñez y su evolución para enlazarlo con un destino y una meta históricos.

\section{Rol del pediatra en relación con la "pedia- tría del desarrollo y del comportamiento"}

Esta área dedicada al estudio del proceso de maduración tiene tres grandes objetivos generales: a) Promover la salud mental y física de los niños y adolescentes, objetivo compartido entre la familia, el equipo de salud y profesores; b) Realizar un diagnóstico temprano y un tra- 
tamiento efectivo de las alteraciones del desarrollo y/o comportamiento, objetivo realizado a través de la apreciación del diagnóstico efectuado por el médico, no necesariamente especialista, que lo deriva; y c) Estudiar los trastornos en el desarrollo, su prevención y manejo más adecuado, cuya responsabilidad recae en los subespecialistas coordinados por el pediatra "de cabecera".

Se entiende por trastornos del desarrollo todos aquellos cuadros crónicos y de inicio precoz que tienen en común la dificultad en la adquisición de habilidades motoras, de lenguaje, sociales o cognitivas que provocan un impacto significativo en el progreso del desarrollo de un niño (Academia Americana de Pediatría, 1994).

En estas condiciones, es deber del pediatra encauzar correctamente las actividades tendientes a minimizar el impacto de estas alteraciones en la evolución del niño. Es decir, el pediatra es el intermediario que debe justipreciar el diagnóstico (efectuado en la práctica por el médico de atención primaria), debe derivar adecuada y oportunamente al especialista correspondiente, y debe ser quien resuma y evalúe el impacto de las intervenciones individuales de cada especialista sobre el menor afectado.

\section{Rol del pediatra en la atención primaria}

El pediatra debe ser el responsable de un equipo multidisciplinario de salud que brinda atención a los niños a lo largo de su ciclo vital, por cuanto su formación y experiencia le brinda los conocimientos, habilidades y destrezas para la solución de los problemas de salud infantil y del adolescente. Este enunciado ideal tiene varias consideraciones:

a) Los programas de formación de especialistas en Pediatría se realizan en su mayor tiempo en actividades intrahospitalarias y con enfermos complejos.

b) Las áreas de promoción, prevención, el enfoque biopsicosocial y la pediatría del desarrollo y del comportamiento son menos enfatizadas durante la formación.

c) Los especialistas se ven poco atraídos por las actividades del nivel primario de atención por la falta de capacidad resolutiva (restricciones en recursos de laboratorio, apoyo diagnóstico y terapéutico), limitacio- nes en las oportunidades de perfeccionamiento, falta de estímulos para carrera funcionaria, entre otros.

d) Los especialistas en Pediatría optan después, por una especialidad derivada que no tiene cabida en el actual sistema de atención primaria.

La atención del adolescente con toda su problemática y con sus patologías propias, debería ser una prioridad, haciendo hincapié en embarazo, salud mental, adicciones y trastornos de la alimentación.

En el actual sistema de atención el pediatra debería además ejercer un rol de filtro o tamizaje que permita evaluar la pertinencia de las derivaciones a especialistas o subespecialistas pediátricos efectuadas por el médico dedicado a niños en la atención primaria y fijar criterios de referencia y contra-referencia que faciliten el accionar de médicos no pediatras que atienden niños.

\section{Atención primaria en manos de médicos extranjeros en forma mayoritaria}

Esta es una realidad difícil de evitar dado el escaso estímulo al médico de Atención Primaria. El exceso de egresados de diferentes universidades, el aumento de oferta de postgrado por parte de universidades insuficientemente $o$ no acreditadas, la mayor relevancia que adquiere la atención ambulatoria con el GES con el consecuente debilitamiento de la actividad pediátrica intrahospitalaria, y el menor peso de la actividad privada ( $8 \%$ de los cotizantes) hacen prever que en el mediano (o corto) plazo, esta realidad se irá revirtiendo al entrar a competir el médico egresado nacional por los cupos de la atención primaria.

Se requiere la implementación de un sistema de actualización permanente, nivelación y reacreditación al médico formado en el extranjero, enfatizando en nuestra realidad epidemiológica y cultural, y de un sistema de estímulos para los médicos recién egresados para trabajar en la atención primaria que corrija las posibles causales del desinterés expresadas previamente (mayor resolutividad, facilidades de perfeccionamiento, carrera funcionaria, etc). 


\section{Formación de recursos humanos}

La Sociedad Chilena de Pediatría no tiene influencia ni relación directa con los centros formadores universitarios, ni sobre los programas de pregrado o postítulo. Puede ofrecer apoyo financiero para programas de formación. Brinda a lo largo del país, a través de sus filiales regionales, ramas de especialidad derivada y comités multidisciplinarios, numerosas actividades de capacitación y perfeccionamiento permanente mediante cursos, jornadas, encuentros con especialistas y congresos. Dispone de sus medios de difusión pública como la Revista Chilena de Pediatría, su página web www. sochipe.cl y el magazín Estetoscopio como vehículos de actualización, difusión de información, motivación y puesta en discusión de temas prioritarios. El Congreso Chileno de Pediatría, que se realiza anualmente, tiene alta convocatoria. Con el Ministerio de Salud hemos trabajado en la elaboración de documentos, publicaciones e integrado comisiones en algunas áreas como nutrición, neonatología, cuidados intensivos, inmunizaciones, maltrato infantil.

Parece necesario que los médicos accedan a una preparación de excelencia y posteriormente tengan la posibilidad de trabajar en un lugar que cuente con la infraestructura necesaria $\mathrm{y}$ con los recursos humanos correspondientes.

Los médicos recién egresados deberían tener entrenamiento en consultorios de atención primaria (generales de zona urbanos) o ir a desempeñarse profesionalmente, por un período variable, a lugares alejados o con déficit de atención médica y luego regresar a algún centro de salud en donde se pueda recibir instrucción en forma de becas de especialidad o estadías de perfeccionamiento (generales de zona y ciclo de destinación).

\section{Reforma de salud}

Las atenciones GES deben tener asegurada la cobertura y contar con los medios que sean necesarios y que esto no vaya en desmedro de las otras patologías. También debe existir una preocupación preferencial por los niños con necesidades especiales de atención, con programas y personal entrenado para hacer prevención. a) Impacto de la reforma de salud (patologías GES) en la salud infantil

El efecto principal es el de asegurar plazos en patologías que, de acuerdo a los estudios previos, tienen mayor impacto en las capacidades y autonomía del ser humano en su ciclo vital. Desde ese punto de vista, el impacto debería medirse a través de indicadores de salud, con énfasis en discapacidades y a largo plazo.

Tal vez el mayor problema es que se circunscribe a aspectos puntuales del desarrollo infantil y apelan a procedimientos de atención simplificados y de bajo costo que no siempre son los que la niñez requiere, con lo cual no representan soluciones de fondo sino más bien medidas paliativas que eluden las respuestas fundamentales y que, sin embargo, pasan por efectivas y valederas al enfatizar en aspectos de tratamiento, y no asegura ninguna política preventiva; es decir, por un lado se refuerza la Atención Primaria en su rol terapéutico, sin embargo, no se refuerza su rol de Prevención Primaria.

No debe descuidarse las patologías no GES, ni hacer una discriminación negativa, sobretodo si esta discriminación está afectada por términos económicos ("valor" del paciente AUGE versus el no AUGE), lo que denigra la acción de salud, al equipo de salud y por supuesto, al propio paciente.

Finalmente, hay un aspecto de reforma de los programas de educación en salud a considerar en el nuevo escenario de la Salud Pública.

\section{b) Requerimientos de atención de niños con enfermedades crónicas versus ofertas de servicios en el nuevo modelo de atención}

La Sociedad Chilena de Pediatría no dispone de información centralizada sobre la demanda de atención de niños con necesidades especiales de atención ni la oferta de servicios y recursos para satisfacer las necesidades planteadas en la reforma de salud. El Comité de Enfermedades Crónicas posee información sobre enfermos crónicos atendidos en diferentes centros hospitalarios que deberá ser actualizada. Sin embargo, solamente los niños con discapacidad neurológica representan, en cortes transversales, cerca del $30 \%$ de los niños 
hospitalizados. Si a eso se le suman las alteraciones más sutiles del desarrollo, el panorama es complejo, correspondiendo a una realidad creciente. Indudablemente, estos niños necesitan un tratamiento interdisciplinario, coordinado por un pediatra a cargo, ubicado en la atención primaria, es decir, lo más cercano a donde vive el paciente, lo que implica modificaciones estructurales de los consultorios así como aumentar el número de centros de salud capacitados. Actualmente, un porcentaje muy elevado de estos niños es atendido por fundaciones que cuentan con escaso aporte fiscal, dependen mucho de aportes voluntarios y de la caridad, lo cual representa una vulnerabilidad importante en su continuidad. Desde el enfoque de la pediatría del desarrollo y del comportamiento, el grupo de niños con este tipo de alteraciones corresponde a un problema de salud importante en términos de autonomía y autovalencia.

\section{c) Suficiencia de recursos para la atención de enfermos crónicos y patologías GES}

De lo anterior, podría inferirse que existe una insuficiencia de recursos en la atención de pacientes portadores de enfermedades crónicas, y que la brecha con los niños con patología GES es de una amplitud preocupante, aunque con tendencia a estrecharse. La falta de recursos aparece tanto en infraestructura como en profesionales, médicos y de colaboración, con suficiente capacitación para hacer sustentable el funcionamiento adecuado.

La adecuación del sistema educativo también está desfasada, y los conceptos de postgrado en pediatría del desarrollo y comportamiento recién están siendo incorporados en los currículos universitarios.

La Sociedad Chilena de Pediatría es integrante de la Asociación de Sociedades Científicas Médicas (ASOCIMED) donde hemos analizado y discutido la distribución de especialistas y los mecanismos de certificación.

\section{Expresión del potencial genético, visión holística y rol de la Sociedad Chilena de Pediatría}

Para la pediatría, es de particular importancia el concepto de que las sociedades humanas están estructuradas en su origen y evolución por una clase de información diferente y superior, "el psiquismo consciente o humano". El desarrollo humano es, en otras palabras, la reproducción y reelaboración de ese psiquismo consciente en cada uno de sus miembros, de acuerdo al contexto social en el que se desenvuelven. La personalidad no es más que la expresión individualizada y singular de ese psiquismo humano. La relevancia de la pediatría radica, precisamente, en que asiste, vigila, protege y promueve la formación de esa personalidad, procurando que tal proceso se desenvuelva en las condiciones más favorables a ésta. Esto es, que en cada ser humano se reproduzcan las conductas, actitudes y valores genuinamente humanos. Desde esta óptica, el pediatra es un modelador de la personalidad y tiene que ser creativo para poner al alcance de los niños, la familia y la comunidad, pautas y modelos de convivencia saludables, constructivos y solidarios.

En todos estos aspectos son los agentes educacionales quienes tendrían responsabilidades. La orientación debe partir desde la enseñanza básica con programas que estimulen el amor al conocimiento y no el estímulo a la competencia, que estimulen las ansias de saber por sobre la preparación para un buen puntaje, y el mérito del establecimiento en desmedro de la salud mental del educando. Esta situación es más acentuada en la enseñanza media. La actitud publicitaria de designar como los mejores colegios en términos de los puntajes obtenidos en las evaluaciones correspondientes, sin interesarse en indagar en los aspectos detrás de una nota, resalta el interés por la superficialidad por sobre los cimientos. Además, el estado premia o castiga de acuerdo a estos mismos parámetros sin establecer equivalencias biosociales entre los establecimientos.

Las sociedades científicas tienen como uno de sus propósitos fundamentales la educación continua, la que, naturalmente, debe estar en consonancia con las políticas de Salud Pública del país. Sin embargo, la opinión de las sociedades científicas escasamente se toma en consideración para estos propósitos, por lo que la injerencia en la toma de decisiones es marginal.

En las propuestas de acciones deberían integrarse la visión política-económica con la vi- 
sión social-humanista, para representar los cambios necesarios. Específicamente, la Sociedad Chilena de Pediatría debería asumir un rol colaborador de las políticas orientadas a fomentar la visión desde la pediatría del desarrollo y del comportamiento, proponiendo actividades, como ya lo ha hecho, y patrocinando o avalando a quienes hagan propuestas en este sentido.

Los cambios curriculares en esta dirección son una realidad desde los años 70-80 en el mundo, abarcando estos aspectos en forma longitudinal en los postgrados de pediatría; pero el agente de cambio debe ser superior, estatal, con políticas multisectoriales que permitan entregar a la familia apoyo para la prevención de los trastornos del comportamiento, ayuda para su oportuno diagnóstico y rehabilitación eficiente para recuperar o limitar las incapacidades físicas o sociales-culturales que, en definitiva, marginan al futuro adulto de expresar sus capacidades. Esto implica agregar en los programas de residencias de pediatría, los aspectos de educación, que prácticamente se ignoran en el ámbito médico y se reserva para los profesionales de enfermería y de colaboración. El pediatra no podrá cumplir su rol de trascendencia, ni siquiera un rol mínimo a favor del desarrollo infantil, sin desplegar su esencia pedagógica: el pediatra educa. En pediatría se cumple muy bien el postulado de Jagger "la curación por la educación". El pediatra es un educador por excelencia: siempre se dirige a los padres, a la comunidad y también al niño, para mostrar los procedimientos y formas de prevenir la enfermedad, de lograr un adecuado crecimiento y desarrollo infantil. Es cierto que la formación actual no nos prepara para diseñar la educación del niño, pero nos corresponde, de manera fundamental, apoyar a la familia a conducir al niño y contribuir a hacerlo hombre, enriquecer su formación y fomentar su realización dentro de la sociedad del futuro.

La visión holística requiere incorporar la mirada sobre el ambiente en que se desarrolla el niño, sector que ha estado restringido a la mirada de educadores y psicólogos y sólo en forma tangencial de los médicos pediatras, quienes nos centramos más en los aspectos biológicos del problema. Las sociedades científicas, junto a las universidades, deben liderar la investigación en los problemas que afectan a los niños, lo que permitirá una mejor comprensión del desarrollo infantil, permitiendo una visión multidisciplinaria y un marco de acción que integre factores biológicos y psicosociales, optimizando la utilización de los recursos humanos, económicos y sociales disponibles en nuestra sociedad. La unificación del conocimiento sobre el desarrollo del niño influirá en la enseñanza, en la atención y en la investigación.

Hacemos propicia esta oportunidad para poner a disposición del Consejo Asesor nuestra plataforma institucional para colaborar y llevar a cabo acciones coordinadas con las autoridades, organismos y ministerios en todos los temas de interés y competencia mutuas.

\section{Referencias}

1.- Schonhaut BL: El rol de la Pediatría del Desarrollo en el control de salud integral: hacia la Pediatría del siglo XXI. Rev Chil Pediatr 2004; 75: 9-12.

2.- De la Barra F, Toledo V, Rodríguez J: Estudio de salud mental en dos cohortes de niños escolares de Santiago occidente. I. Prevalencia y seguimiento de problemas conductuales y cognitivos. Rev Chil NeuroPsiquiat 2002; 40: 9-21.

3.- Pascucci Mc, Lejarraga H, Kelmansky D, et al: Validación de la prueba nacional de pesquisa de trastornos de desarrollo psicomotor en niños menores de 6 años. Rev Chil Pediatr 2004; 75: 75-6.

4.- Lavados P, Gómez V, Sawada M, Chomali M, Álvarez M: Diagnósticos neurológicos en la atención primaria de salud en Santiago, Chile. Rev Neurol 2003; 36: 518-22.

5.- Werner D: Más allá de la pediatría: la salud y la supervivencia de los niños en situación desventajosa. Pediatrics (Edición en español) 1993; 35: 218-20.

6.- Avaria M: Pediatría del desarrollo y comportamiento. Rev Ped Elec 2005; 2: 88-91. 